\title{
Size-scaling experiment in a two-dimensional electron gas using an inhomogeneous magnetic field due to a superconducting gate
}

\author{
A.K. Geim " , S.V. Dubonos ${ }^{\text {b.* }}$, I.V. Grigorieva a , S.J. Bending ${ }^{c}$ \\ - High Field Magnet Laboratory, University of Nifmegen, 6500 GL Nijmegen, The Netherlands \\ D Institute of Microelectronics Technology, 142432 Chernogolovka, Russia \\ - School of Physics, University of Bath, Bath BA2 7AY, England, UK
}

Received 21 June 1995; accepted for publication 12 August 1995

\begin{abstract}
We have used a superconducting gate on the top of a two-dimensional electron gas (2DEG) to partition an external magnetic field into a distribution of submicron flux tubes (vortices) and measure the weak localisation contribution to the 2DEG conductance versus spatial separation of the vortices. It is found that the effect of a vortex extends far beyond its actual size. The experiment is discussed in terms of size scaling of the quantum conductance. The measured minimum size of an independent region of a 2DEG is in agreement with the phase-breaking length of electrons as deduced from the weak localisation magnetoresistance.
\end{abstract}

Keywords: Electrical transport; Heterojunctions; Quantum effect; Superconductor-semiconductor thin film structures

\section{Introduction}

There has been a growing interest in a hybrid superconductor-two-dimensional electron gas (2DEG) system in which an extremely inhomogeneous magnetic field created by a type II superconducting gate is projected down onto a $2 \mathrm{DEG}$ below. An applied magnetic field is segregated within and near the superconductor into a distribution of magnetic-flux tubes (vortices) with a characteristic diameter $d$ of only $\sim 0.2 \mu \mathrm{m}$ (see Fig. 1). A number of novel phenomena have been reported for this system, including non-local weak localisation [1], conductance fluctuations due to a single vortex in a mesoscopic conductor [2], and diffrac-

"Corresponding author. Fax: + 31243652440 e-mail: geim@scikun.nl. tion of 2D electron waves at vortices [3]. A point of fundamental interest here is the fact that characteristic relaxation lengths in a 2DEG can be much longer than the size of vortices, in which case the transport is essentially non-local.

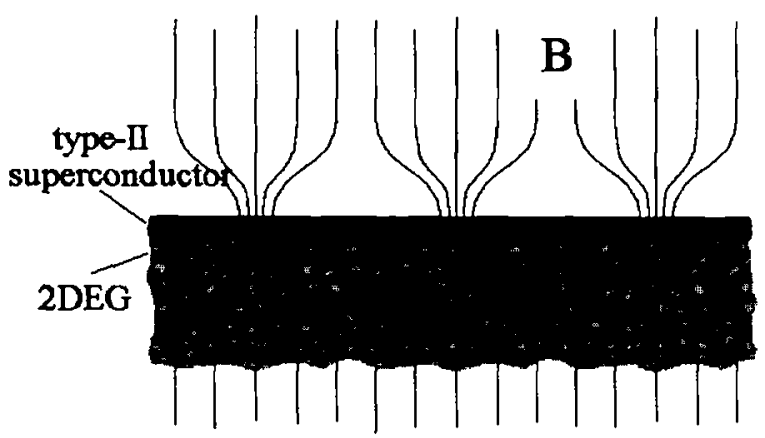

Fig. 1. The 2DEG-superconductor hybrid system. 
In this work, superconducting vortices have been employed to study size-scaling in zero-field resistivity of a 2DEG. For a vortex concentration $N=$ $L^{-2}$, each $L \times L$ square of the 2DEG is "earmarked" by a vortex $(d \ll L)$ which slightly changes the resistance of the square. As the vortex concentration increases, the 2DEG effectively becomes divided into smaller and smaller squares. Up to a certain concentration of vortices, the total resistance of the 2DEG is found to be proportional to the number of vortices. However, as the concentration is increased further, the linear dependence breaks down indicating that we have reached the minimum size $L_{\min }(\gg d)$, below which simple scaling is violated. In other words, it is impossible to put a vortex inside the 2DEG (or do anything else with the 2DEG) without changing the properties of the entire region $L_{\min } \times L_{\min }$. We have measured the size of the minimum square for a number of 2DEGs and compared $L_{\text {min }}$ with the phase-breaking length of electrons $L_{\phi}$ extracted from fitting the curves for weak localisation magnetoresistance in a uniform field. It is found that $L_{\min } \approx 2 L_{\phi}$. We note that our experiment appears to be a very close implementation of the wellknown gedanken experiment, where a conducting plane is divided into square of sequentially decreasing sizes. Instead of cutting a macroscopic sample into pieces as the size-scaling experiment infers, we have effectively divided the 2DEG by tagging its parts with vortices. Thus, we have been able to give a concrete physical significance to $L_{\phi}$ in terms of our vortex mesh size, in stark contrast to all previous work where $L_{\phi}$ only enters as a fitting parameter in theoretical models.

\section{Experimental}

Our experimental system is shown schematically in Fig. 1. A lead superconducting film $(\sim 0.1 \mu \mathrm{m}$ in thickness) was deposited on the top of GaAlAs/GaAs heterostructures with a $2 \mathrm{DEG}$ at distances of $60-80 \mathrm{~nm}$ from the surface. Lead films of these thicknesses are type II superconductors and have a very small value of magnetic-field penetration depth $\lambda \approx 60 \mathrm{~nm}$ at $1.3 \mathrm{~K}$. Taking into account spatial broadening of vortices when they emerge from the superconductor, we estimate $d \approx 0.2 \mu \mathrm{m}[1-3]$. The vortex concentration in the 2DEG is controlled by the external magnetic field $B$ as $N=B / \phi_{0}$ where $\phi_{0}=h / 2 e$ is the superconducting flux quantum. Vortices in the 2DEG remain well-separated in applied magnetic fields up to $100 \mathrm{G}$. At larger fields, the distance between individual vortices, $L(\mu \mathrm{m})=\left(\phi_{0} / B\right)^{1 / 2} \approx 5[B(\mathrm{G})]^{-1 / 2}$, decreases to a value comparable with the vortex diameter, and magnetic fields due to adjacent vortices overlap strongly. Consequently, the field inhomogeneity rapidly smears out, even though the lead gate remains superconducting up to $\sim 1 \mathrm{kG}$. In this paper we discuss results for three heterostructures with electron concentrations of $\sim 5 \times 10^{15} \mathrm{~m}^{-2}$ and mobilities 3, 3.7 and $5.9 \mathrm{~m}^{2} / \mathrm{V} \cdot \mathrm{s}$. The relatively low mobility of the 2DEG has been chosen intentionally to avoid the ballistic effects discussed in Ref. [3]. These effects give rise to an extra resistivity due to scattering of electrons at the vortices, but this contribution is negligibly small for all data presented below. For further experimental details we refer to Refs. [1-3].

\section{Results and discussion}

Figs. $2 \mathrm{a}$ and $2 \mathrm{~b}$ show the magnetoresistance of one of the samples in magnetic fields below $25 \mathrm{G}$ at temperatures of 1.3 and $4.2 \mathrm{~K}$. For brevity, the experimental data are presented directly in terms of $2 D E G$ conductivity $\Delta \sigma$ and in units of the conductance quantum $e^{2} / h$. In the uniform magnetic field (solid curve) the sample exhibits a conventional negative magnetoresistance due to suppression of the time-reversal contribution to electron interference [4-6]. This magnetoresistance is well described by Kawabata's formulae for weak localisation (dashed curve), which is applicable for our samples with a relatively long ballistic mean free path $l \approx 0.5 \mu \mathrm{m}$ comparable with $L_{\phi}$ [4]. The fit allows us to find $L_{\phi}$. We note that the Hikami-Larkin-Nagaoka theory [5], valid for $l \ll L_{\phi}$, gives us a slightly worse fit and yields somewhat smaller values of $L_{\phi}$ (typically $20 \%$ smaller). The symbols in Fig. 2 correspond to the case of the non-uniform field created by the superconductor. The initial linear increase in conductiv- 


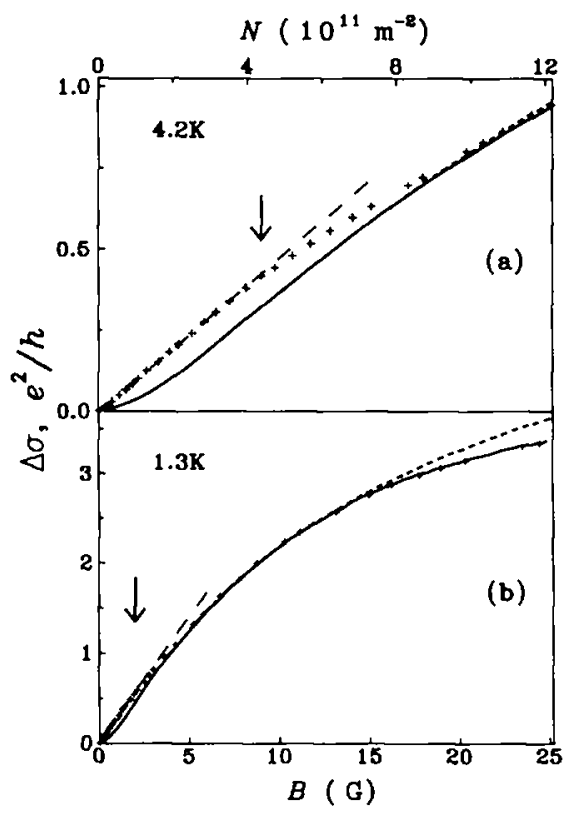

Fig 2. Magnetoconductivity of the $2 D E G$ in uniform magnetic field (solid curves) and in the distribution of vortices (crosses) at temperatures of (a) 4.2 and (b) $1.3 \mathrm{~K}$. The electron mobility is $3 \mathrm{~m}^{2} / \mathrm{V} \cdot \mathrm{s}$ and the electron concentration is $5.3 \times 10^{15} \mathrm{~m}^{-2}$. The short-dashed curves are the best fit to the theory of weak localisation in homogeneous field. The long-dashed lines indicate the linear vortex magnetoresistance in low fields.

ity of the 2DEG is due to the linear increase in vortex concentration when $B$ increases. Note that the same field $B$ influences the 2DEG much more strongly when the magnetic flux is segregated into a distribution of submicron tubes. This enhancement of the magnitude of the weak localisation magnetoresistance has been reported earlier [1] and is discussed in detail in Ref. [6].

Below, we will concentrate on another striking feature in the additional resistivity due to the presence of vortices in the 2DEG. For the plotted interval of vortex concentrations in Fig. 2, magnetic fields due to adjacent vortices do not overlap. Nevertheless, the curves for the uniform magnetic field and the inhomogeneous field of vortices converge when the vortices are as far as several $\mu \mathrm{m}$ apart. At lower temperatures, the difference between the two cases disappears at even lower fields, i.e. for larger vortex separations (see Fig. 2). The observed behaviour indicates that a vortex

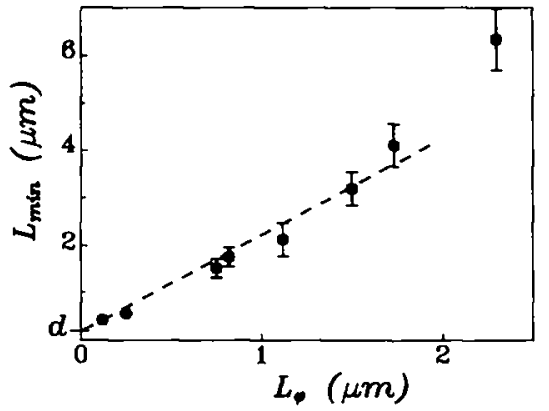

Fig. 3. Dependence of the size of a 2DEG region affected by a vortex $\left(L_{-\infty}\right)$ on the phase-breaking length of electrons $\left(L_{\phi}\right)$ deduced from the weak localisation magnetoresistance in the uniform field.

suppresses the weak localisation in the 2DEG at distances far beyond the region where its magnetic field is present. When the extended regions of the 2DEG affected by adjacent vortices overlap, the dependence $\Delta \sigma \propto N$ breaks down. We define $N_{\min }$ as the vortex concentration when deviations from the linear dependence exceed a level of the scatter in the experimental data. In Fig. 2 such concentrations are shown by arrows. The value of $N_{\min }$ yields the size of an independent unit of the 2DEG as $L_{\min }=N_{\min }^{-1 / 2}$. Fig. 3 plots $L_{\min }$ versus $L_{\phi}$ using data for all three 2DEGs at temperatures of 1.3 and $4.2 \mathrm{~K}$. We have also included here the results from Ref. [1] for non-local weak localisation in thin Bi films. The dotted line in Fig. 3 shows the best linear fit to the experimental data, which yields $L_{\min } \approx 2 L_{\phi}+d$. This dependence shows that the influence of vortices extends over the distance $\sim L_{\phi}$ outside the region of the magnetic field, in agreement with the nature of the effect.

We note that the observed spatial enhancement of the effect of vortices is not due to the vector potential, which of course extends outside the region of the magnetic field. Weak localisation is due to the constructive interference on timereversal trajectories and the superconducting flux quantum $h / 2 e$ cannot destroy it since the acquired phase shift is equal to $2 \pi$ [4-6]. Therefore, only those trajectories which pass through the region of the magnetic field, and hence envelop a part of the superconducting flux quantum, are influenced by vortices. The origin of the observed spatial enhancement lies in the statistics of diffusion in 
two dimensions, where a diffusive electron trajectory circles any point in space an infinite number of times. Although in reality this divergence is suppressed due to the finite phase-coherence length, the statistics dictate that every trajectory within a phase-coherent sample can pass through a vortex located inside [6]. The influence of vortices becomes non-additive (adjacent vortices begin to "feel" the presence of each other) when the phase-coherent trajectories pass through more than one vortex.

Finally, we note that several features in the observed behaviour remain to be explained. First, it is not clear whether the coefficient 2 in the empirical expression for $L_{\min }$ is meaningful or relates to our definition of $N_{\min }$. We speculate that electrons with the highest probability of interacting with two vortices will have their origins midway between them. Thus the scaling behaviour would be expected to start to break down when such an electron has its origin at the distance $L_{\phi}$ from two adjacent vortices, or when the centre-to-centre vortex spacing is $2 L_{\phi}+d$. The second surprising feature is a very small interval between $N_{\min }$ and the vortex concentration where the curves for the uniform and non-uniform fields converge completely (see Fig. 2). It is also worth mentioning that we have not seen the spatial extension of the effect of vortices for the case of ballistic scattering of $2 \mathrm{D}$ electrons at vortices [3]. In that experiment, the vortex resistivity disappeared only when the field inhomogeneity smeared out.

\section{Conclasion}

We have realised the size-scaling gedanken experiment for the case of weak localisation.
Placing a number of vortices inside a macroscopic 2DEG we effectively divide it into a number of smaller samples, each ear-marked by a single vortex. As the vortex separation is decreased, a distinct change in the effect of vortices on the 2DEG is observed, indicating the size of the minimum independent region in the 2DEG. This size is found to be of the order of the phase-breaking length of electrons $L_{\phi}$ deduced from the weak localisation magnetoresistance. Although the importance of this scale has followed from the theory and experiments on weak localisation and mesoscopics, the gedanken experiment has never been realised, primarily because of the problem of electrical contacts to small quantum devices.

\section{References}

[1] A.K. Geim, JETP Lett. 50 (1989) 389; S.J. Bending, K.v. Klitring and K. Ploog, Phys. Rev. Lett. 65 (1990) 1060.

[2] A.K. Geim, V.I. Falko, S.V. Dubonos and I.V. Grigorieva, Solid State Commun. 82 (1992) 831.

[3] A.K. Geim, S.J. Bending and I.V. Grigorieva, Phys. Rev. Lett. 69 (1992) 2252;

A.K. Geim, S.J. Bending, I.V. Grigorieva and M.G. Blamire, Phys. Rev. B 49 (1994) 5749.

[4] A. Kawabata, J. Phys. Soc. Jpa. 53 (1984) 3540.

[5] S. Hikami, A.I. Larkin and Y. Nagaoka, Prog. Theor. Phys. 63 (1980) 707.

[6] J. Rammer and A.L. Shelankov, Phys. Rev. B 36 (1987) 3135;

S.J. Bending and A.K. Geim, Phys. Rev. B 46 (1992) 14912. 